\title{
Comparison of doses to the rectum derived from treatment planning system with in-vivo dose values in vaginal vault brachytherapy using cylinder applicators
}

\author{
Emmanuel Oyeyemi Oyekunle, PhD!, Rachel Ibhade Obed, PhD², Bidemi ldayat Akinlade, PhD³ Atara Ntekim, MD³ \\ 'Department of Radiotherapy, University College Hospital (UCH), ${ }^{2}$ Department of Physics, University of Ibadan, ${ }^{3}$ Department of Radiotherapy, \\ College of Medicine, University of Ibadan, Ibadan, Nigeria
}

\begin{abstract}
Purpose: In-vivo measurements to determine doses to organs-at-risk can be an essential part of brachytherapy quality assurance (QA). This study compares calculated doses to the rectum with measured dose values as a means of QA in vaginal vault brachytherapy using cylinder applicators.

Material and methods: At the Department of Radiotherapy, University College Hospital (UCH), Ibadan, Nigeria, intracavitary brachytherapy (ICBT) was delivered by a GyneSource high-dose-rate (HDR) unit with ${ }^{60} \mathrm{Co}$. Standard 2D treatment plans were created with HDR basic 2.6 software for prescription doses 5-7 Gy at points 5 mm away from the posterior surface of vaginal cylinder applicators (20, 25, and $30 \mathrm{~mm}$ diameters). The LiF:Mg, Ti thermoluminescent dosimeter rods $(1 \times 6 \mathrm{~mm})$ were irradiated to a dose of $7 \mathrm{~Gy}$ on Theratron ${ }^{60} \mathrm{Co}$ machine for calibration purpose prior to clinical use. Measurements in each of 34 insertions involving fourteen patients were performed with 5 TLD-100 rods placed along a re-usable rectal marker positioned in the rectum. The dosimeters were read in Harshaw 3500 TLD reader and compared with doses derived from the treatment planning system (TPS) at $1 \mathrm{~cm}$ away from the dose prescription points.

Results: The mean calculated and measured doses ranged from 2.1-3.8 Gy and 1.2-5.6 Gy with averages of 3.0 $\pm 0.5 \mathrm{~Gy}$ and $3.1 \pm 1.1 \mathrm{~Gy}$, respectively, for treatment lengths $2-8 \mathrm{~cm}$ along the cylinder-applicators. The mean values correspond to $48.9 \%$ and $50.8 \%$ of the prescribed doses, respectively. The deviations of the mean in-vivo doses from the TPS values ranged from -1.9 to 2.1 Gy with a $p$-value of 0.427 .

Conclusions: This study was part of efforts to verify rectal dose obtained from the TPS during vaginal vault brachytherapy. There was no significant difference in the dose to the rectum from the two methods of measurements.
\end{abstract}

Key words: brachytherapy, rectum, cervical cancer, in-vivo dose, vaginal cylinder, vaginal vault.

\section{Purpose}

In spite of the fact that intracavitary brachytherapy (ICBT) has been used in the treatment of cervical cancer for more than 20 years, the potential for increased risk of late complications, especially in the rectum is a major concern [1]. Vaginal cylinder is an alternative treatment tool to tandem-ring or tandem-ovoid applicators in cervical cancer patients who had undergone hysterectomy. Similarly, it is used to treat vaginal vault after hysterectomy following endometrial cancer. In these cases, the uterine canal is no longer available for placement of the intra-uterine tandem (IUT). As such, rather than the use of ring or ovoids appli- cators, which cannot adequately treat the distal part of the vaginal vault, vaginal cylindrical applicators attached to a single central channel or multiple channels are often used. Cervical cancer is the most common indication for brachytherapy in most developing countries and highdose-rate (HDR) brachytherapy equipment are capable of treating large number of patients. Hence, HDR BT is recommended for developing countries with high incidence of the disease [2]. The HDR brachytherapy technique in Nigeria commenced in 2008 at the University College Hospital (UCH), Ibadan. At $\mathrm{UCH}$, our records indicated that $85 \%$ of patients receiving ICBT use the tandem-ring applicator, while the proportion requiring vaginal cylin-
Address for correspondence: Emmanuel Oyeyemi Oyekunle, PhD, Medical Physics Unit, Department of Radiotherapy, University College Hospital (UCH), Queen Elizabeth Road, Mokola, Ibadan, Nigeria, phone: +2348023262120, e-mail: emmanueloyekunle.eo@gmail.com,yemm7@yahoo.com
Received: 15.05.2015

Accepted: 27.10.2015

Published: 30.12.2015 
der is about $15 \%$. Unlike the three-channel ring/ovoidIUT applicators, in-vivo dosimetry is uncommon for single-channel vaginal cylinders, since dose contribution to organs-at-risk (OARs), such as the rectum, is mainly due to the cylinder. In tandem-cylinder applications, dose prescription points are usually at $5 \mathrm{~mm}$ away from the posterior surface of the cylinder. A study by Demanes et al. [3] showed that the multichannel vaginal cylinder allows much better dose control than the single-channel type, as the former achieved lower rectal doses by $15 \%$, when compared to the latter.

In-vivo dosimetry is a useful tool for evaluating the doses to OARs, such as the rectum, as part of quality assurance (QA) for assessing the HDR technique including the treatment planning system (TPS). Thermoluminescent dosimeters (TLDs) and semiconductor diodes have been commonly used by many researchers for in-vivo dosimetry $[4,5,6,7]$. These dosimeters are used for point dose measurements and hence suffer from the usual drawback that the measured dose may not always represent the clinically significant dose to OARs. Therefore, measurements at several points may be needed. This study was designed to compare doses to the rectum derived from the TPS with in-vivo dose values using TLDs, as a means of QA in ICBT with cylinder applicators at the pioneer HDR brachytherapy center in Nigeria.

\section{Material and methods}

This was a prospective study carried out among cervical and endometrial cancer patients who had hysterectomy, and were booked to have HDR brachytherapy to the vaginal vault at the University College Hospital, Ibadan, Nigeria. Approval for the study was obtained from the institution's Ethical Review Committee and the patients gave consent for their participation. Vaginal vault brachytherapy was carried out using single-channel vaginal cylinders and in-vivo dosimetry was done using TLD100 rods. Data analysis in this study was performed using GraphPad Prism 6 statistical software (San Diego, USA).

\section{TLD-100 rods and calibration}

Tissue equivalence characteristic of detector, small size, and its affordability informed the choice of TLDs among other possible detectors. The TLDs, Li:Mg, Ti rods

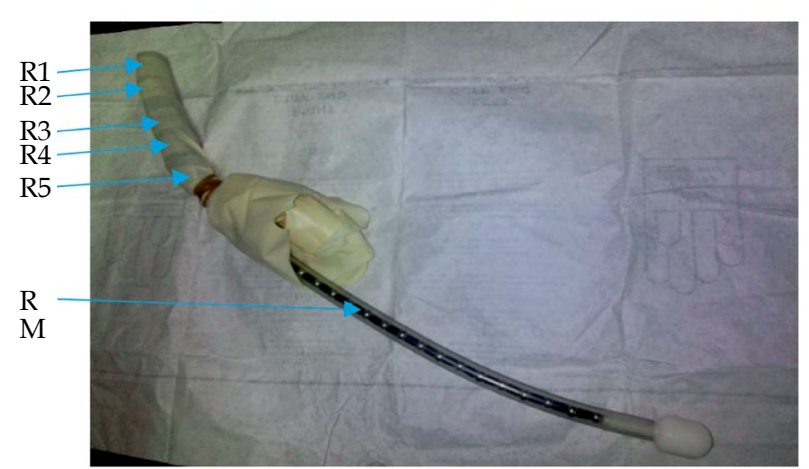

Fig. 1. Re-usable rectal marker (RM) bearing five TLDs (for rectal dose points, R1-R5) enclosed in a finger of a glove
(6 mm length, $1 \mathrm{~mm}$ diameter) used for this study were procured from Harshaw, Ohio, USA. The dimensions of the rod type were considered more suitable for in-vivo study as in this work. The dosimeters were annealed in a TLD oven at the Center for Energy Research and Development (CERD), Obafemi Awolowo University, Ile-Ife, Nigeria. They were initially irradiated with a dose of 1 Gy using Theratron 780C telecobalt machine (MDS Nordian, Canada, Inc.) at the Radiotherapy Center of EKO Hospital, Lagos, Nigeria. The telecobalt machine was pre-calibrated with a Farmer ionization chamber: NEL 2581, serial no. $837\left(0.6 \mathrm{~cm}^{3}\right)$ and the associated electrometer: 2570/1 Farmer, serial no. 967. The TLDs were placed on the surface of a $30 \times 30 \times 17.6 \mathrm{~cm}^{3}$ polymethylmethacrylate (PMMA) phantom during irradiation with a field size of $20 \mathrm{~cm} \times 20 \mathrm{~cm}$. The dosimeters were kept within the center of the radiation beam. A radiographic film was used to check the dose uniformity of the absorbed dose delivered.

The irradiated TLDs were read using a Harshaw 3500 reader. Five calibration (golden) dosimeters with best responses (values $\pm 2 \%$ of $1 \mathrm{~Gy}$ ) were determined and selected. All the TLDs were re-annealed, with the golden dosimeters separately identified. The entire dosimeters were irradiated with a dose of $7 \mathrm{~Gy}$ (typical of brachytherapy dose) using the same external beam radiation therapy (EBRT) unit above. The five golden TLDs were first read and used to calibrate the TLD reader. The other dosimeters were read in turn and the element correction coefficient (ECC) was generated for each according to equation (1).

$$
E C C=\frac{(T L D)}{T L D_{j}}
$$

where $T L D_{j}$ and (TLD) are the individual reading of TLDs and the mean value, respectively.

\section{Measurements with TLD-100}

During the period between June and August 2013, thirty-four ICBT applications using single-channel cylinder applicators were carried out. Fourteen patients with cervical and endometrial cancers who had hysterectomy at $\mathrm{UCH}$ were included in this study. Prior to clinical use, each dosimeter was placed in a small black polythene bag properly labelled for identification after annealing in the TLD oven. Five separate dosimeters were placed at $5 \mathrm{~mm}$ interval on the posterior surface of a flexible rectal marker, which is of $5 \mathrm{~mm}$ thickness. The rods were stabilized using tapes on the surface of the rectal marker. Each ICBT insertion involved the use of five detectors in order to obtain reasonable mean doses, and therefore increase the accuracy of rectal TLD dose measurements. Following the selection and insertion of suitable cylinder applicator size in the vagina, the TLD-100 rods on rectal marker were placed within the rectum. To make it re-useable, the rectal marker was always enclosed in a finger of a hand's glove and held tightly with an elastic rubber (Figure 1). The gloves containing the TLD rods were doubled in order to prevent the TLDs from absorbing body fluids within the cavity. It was then carefully pushed into 

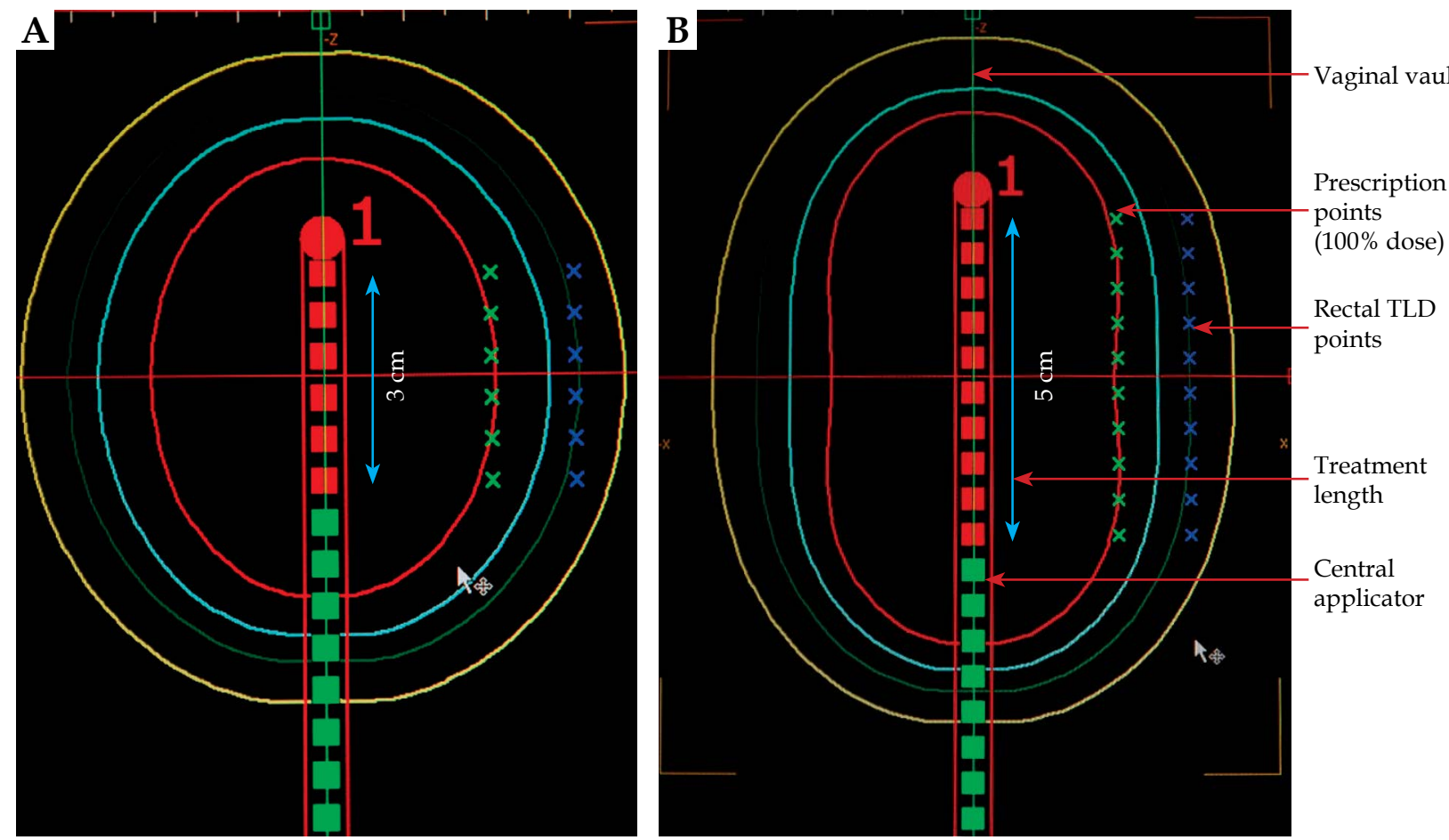

Fig. 2. Variations in dose distributions in two brachytherapy standard plans using $30 \mathrm{~mm}$ central channel vaginal cylinder (CCVC) with different treatment lengths. A) $30 \mathrm{~mm}$ treatment length, B) $50 \mathrm{~mm}$ treatment length

the rectum such that the TLDs were placed posteriorly at $1 \mathrm{~cm}$ distance from the dose prescription points $(5 \mathrm{~mm}$ rectal wall thickness plus $5 \mathrm{~mm}$ diameter of the rectal marker). The entire procedure was under sterile conditions. Before delivery of brachytherapy, a detailed gynecologic examination was performed to evaluate vaginal vault and to determine applicator diameter, as was done in a previous study [8]. Selection of the vaginal cylinder diameter and the treatment length for a given prescribed dose was made after vagina examination. Source loading of dwell points according to the defined treatment length was carried out after the selected cylinder was extracted from the applicator library of our TPS using HDR basic version 2.6. (Bebig, Germany). Standard (2D) plans similar to those shown in Figure 2 were created. In order to derive rectal doses from the TPS, a set of control points (described by coordinates given in Table 1B) was defined at $1 \mathrm{~cm}(5 \mathrm{~mm}$ rectal wall thickness plus $5 \mathrm{~mm}$ diameter of the rectal marker) away from dose prescription points. These points correspond to the position of the TLD rods in-vivo. In principle, a control point corresponds to a dwell point on an applicator. The dwell points used on the TPS at UCH have been set at $5 \mathrm{~mm}$ intervals (step size) and will normally vary with the treatment length along the straight IUT applicator (tandem) attached to the cylinder. Therefore, the total dwell points are 4, 6, $8,10,12$, and 16 for treatment lengths $2,3,4,5,6$, and 8 $\mathrm{cm}$, respectively, involved in this study. Dose prescription points were $5 \mathrm{~mm}$ away from the posterior surface of the cylinder according to the recommendations of the International Commission on Radiation Units and Measurements [9]. The radius of the selected cylinder is in reference to its center. As such, ICBT fraction dose, which varied depending on the disease type, was prescribed at points 15, 17.5, and $20 \mathrm{~mm}$ from applicator midpoints for cylinders 20,25 , and $30 \mathrm{~mm}$ diameter, respectively. The prescribed doses were 15, 19.5, and 21 Gy in 3 fractions delivered on weekly basis, following EBRT of 45 Gy in 22 fractions with Theratron $780 \mathrm{C}$ telecobalt unit. The values calculated by the TPS at the prescription points and the set rectal dose points are presented in Tables $1 \mathrm{~A}$ and $1 \mathrm{~B}$, respectively.

Brachytherapy was delivered immediately following applicator insertion and placement of rectal TLDs using the standard (2D) treatment plans. As no simulation radiographs were obtained, there was no additional dose contribution (from $\mathrm{X}$-rays) to the dosimeters. Thirty four treatment procedures were performed in fourteen patients.

At the end of each treatment, the rectal marker was removed from the patient and the dosimeters were carefully removed. The absorbed dose in the TLD after irradiation, in the form of electrons in the energy traps of the crystal lattice, was retrieved by heating up the dosimeter at a predefined temperature rate of $10^{\circ} \mathrm{C} / \mathrm{s}$ to a maximum temperature $\left(300^{\circ} \mathrm{C} / \mathrm{s}\right)$ in the Harshaw 3500 TLD reader. The mean rectal dose measured with TLD for each insertion was obtained from the five dosimeters at points R1$\mathrm{R} 5$ on the in-vivo marker.

\section{Results}

A total of 34 ICBT insertions was carried out between June and August 2013. The mean calculated doses (TPS) and measured doses (TLD rods) in these brachytherapy applications with the cylinder applicator sizes are pre- 
Table 1.

A) A sample of dose prescription points' details as reported on the treatment planning system (TPS) for a vaginal cylinder $30 \mathrm{~mm}$, treatment length $3 \mathrm{~cm}$ and prescribed dose of $5 \mathrm{~Gy}$

\begin{tabular}{lccccc} 
Idx & X-Pos $[\mathrm{cm}]$ & Y-Pos $[\mathrm{cm}]$ & Z-Pos $[\mathrm{cm}]$ & Dose $[\mathrm{Gy}]$ & Dose \% Rx \\
\hline 1 & 2.00 & 0.08 & -3.05 & 4.71 & 94.2 \\
\hline 2 & 2.00 & 0.07 & -2.55 & 4.98 & 99.6 \\
\hline 3 & 2.00 & 0.07 & -2.05 & 5.02 & 100.4 \\
\hline 4 & 2.00 & 0.07 & -1.55 & 5.03 & 100.6 \\
\hline 5 & 2.00 & 0.07 & -1.05 & 4.98 & 99.6 \\
\hline 6 & 2.00 & 0.06 & -0.55 & 4.71 & 94.2
\end{tabular}

Ctrl Point Group Name: LAR01-01 \# $2.00 \mathrm{~cm}$

$R x=5.00 \mathrm{~Gy}$

Min. dose: $4.71 \mathrm{~Gy}$

Average dose: 4.91 Gy $(98.2 \% R x)$

Max. dose: $5.03 \mathrm{~Gy}$

B) Sample of defined dose control (rectal thermoluminescent dosimeters - TLD) points' details as reported on the treatment planning system (TPS) for a vaginal cylinder $30 \mathrm{~mm}$, treatment length $3 \mathrm{~cm}$ and prescribed dose of 5 Gy

\begin{tabular}{|c|c|c|c|c|c|}
\hline Idx & X-Pos $[\mathrm{cm}]$ & Y-Pos $[\mathrm{cm}]$ & Z-Pos $[\mathrm{cm}]$ & Dose [Gy] & Dose $\% \mathrm{Rx}$ \\
\hline 1 & 3.00 & 0.08 & -3.05 & 2.24 & 44.8 \\
\hline 2 & 3.00 & 0.07 & -2.55 & 2.42 & 48.3 \\
\hline 3 & 3.00 & 0.07 & -2.05 & 2.51 & 50.3 \\
\hline 4 & 3.00 & 0.07 & -1.55 & 2.51 & 50.3 \\
\hline 5 & 3.00 & 0.07 & -1.05 & 2.42 & 48.4 \\
\hline 6 & 3.00 & 0.06 & -0.55 & 2.24 & 44.8 \\
\hline
\end{tabular}

Ctrl Point Group Name: LAR01-01 \# $3.00 \mathrm{~cm}$

$R x=5.00 \mathrm{~Gy}$

Min. dose: 2.24 Gy

Average dose: 2.39 Gy $(47.80 \% R x)$

Max. dose: $2.51 \mathrm{~Gy}$

sented in Table 2. The deviations of the measured from the mean calculated doses varied from - 1.9 to $2.1 \mathrm{~Gy}$. Table 3 shows the comparisons between calculated TPS rectal doses and doses in-vivo with respect to the minimum and maximum values in the thirty-four ICBT insertions. Figure 3 compares the mean calculated rectal dose values with the mean measured (TLD) rectal doses. Average rectal dose calculated and measured dose per ICBT insertion ranged from 2.1-3.8 Gy and 1.2-5.6 Gy with overall mean doses of $3.0 \pm 0.5 \mathrm{~Gy}$ and $3.1 \pm 1.1 \mathrm{~Gy}$, respectively. The combined (calculated and measured) means yielded $48.9 \%$ and $50.8 \%$ of the fractional prescription doses of 7 Gy $(n=16), 5$ Gy $(n=15)$ and 6.5 Gy $(n=3)$. This did not show statistically significant difference $(p=0.427)$.

\section{Discussion}

Dose distribution in ICBT clinical target volume (CTV) with cylinder applicators will depend on treatment length, cylinder diameter, prescribed dose, distance from the first dwell position to the tip of the applicator, and the shape of the distal part of the applicator [10]. The most crucial are the first two factors as the treat- ment length and cylinder diameter. The treatment length, which is determined by the radiation oncologist in the brachytherapy suite will normally vary among patients and sometimes within a given patient from one insertion to another. The applicator diameter will depend on the size of the vaginal cavity such that the cylinder that can be adequately inserted and fitted within the vagina. Physical assessment of the patients by vagina examination during each brachytherapy insertion determined the length of the IUT that was activated with source loading as shown in Figure 2. Treatment lengths along the applicator in this study varied from $2-8 \mathrm{~cm}$. In this study, we adopted a limit of $70 \%$ of the fractional prescription doses to assess the rectal doses measured in-vivo by the TLDs. In $31(91.2 \%)$ and $3(8.8 \%)$ insertions (Table 2), the mean measured doses were within and greater than $70 \%$ of the prescription doses, respectively. The extent (length) of treatment on the applicator increases the dimension of the isodose longitudinally and this will have impact on rectal dosimetry. In-vivo dosimetry in this study has shown that a larger applicator has the tendency to increase dose contribution within the rectum in vagina vault brachytherapy. In our study, the extreme rectal 
Table 2. Comparison of mean in-vivo doses and calculated (treatment planning system - TPS) doses in intracavitary brachytherapy (ICBT) with the cylinder applicator

\begin{tabular}{|c|c|c|c|c|c|c|}
\hline $\mathrm{s} / \mathrm{n}$ & $\begin{array}{l}\text { Cylinder, } \\
\text { diameter } \\
(\mathrm{mm})\end{array}$ & $\begin{array}{l}\text { Treatment, } \\
\text { length }(\mathrm{cm})\end{array}$ & $\begin{array}{l}\text { Prescribed } \\
\text { dose (Gy) }\end{array}$ & $\begin{array}{c}\text { Mean calculated } \\
\text { (TPS) dose at TLD } \\
\text { points (Gy) }\end{array}$ & $\begin{array}{c}\text { Mean measured } \\
\text { (in-vivo) doses (Gy) } \\
\text { (TLD) }\end{array}$ & $\begin{array}{c}\text { Deviations } \\
\text { (calculated - } \\
\text { measured dose) }\end{array}$ \\
\hline 1 & 20 & 4 & 7.0 & 3.1 & 1.9 & 1.2 \\
\hline 2 & 20 & 4 & 7.0 & 3.1 & 2.6 & 0.5 \\
\hline 3 & 20 & 4 & 7.0 & 3.1 & 3.0 & 0.1 \\
\hline 4 & 20 & 4 & 7.0 & 3.1 & 3.2 & -0.1 \\
\hline 5 & 20 & 5 & 5.0 & 2.4 & 4.1 & -1.7 \\
\hline 6 & 20 & 5 & 5.0 & 2.4 & 2.3 & 0.1 \\
\hline 7 & 25 & 2 & 5.0 & 2.1 & 2.6 & -0.5 \\
\hline 8 & 25 & 2 & 5.0 & 2.1 & 2.3 & -0.2 \\
\hline 9 & 25 & 3 & 7.0 & 3.1 & 2.7 & 0.4 \\
\hline 10 & 25 & 3 & 7.0 & 3.1 & 1.6 & 1.5 \\
\hline 11 & 25 & 4 & 7.0 & 3.3 & 1.2 & 2.1 \\
\hline 12 & 25 & 4 & 7.0 & 3.3 & 3.7 & -0.4 \\
\hline 13 & 25 & 4 & 5.0 & 2.4 & 1.3 & 1.1 \\
\hline 14 & 25 & 4 & 5.0 & 2.4 & 1.8 & 0.6 \\
\hline 15 & 25 & 4 & 5.0 & 2.4 & 2.0 & 0.4 \\
\hline 16 & 25 & 4 & 5.0 & 2.4 & 2.4 & 0 \\
\hline 17 & 25 & 5 & 7.0 & 3.4 & 4.1 & -0.7 \\
\hline 18 & 25 & 5 & 7.0 & 3.4 & 4.3 & -0.9 \\
\hline 19 & 25 & 5 & 7.0 & 3.4 & 3.3 & 0.1 \\
\hline 20 & 25 & 5 & 7.0 & 3.4 & 4.9 & -1.5 \\
\hline 21 & 30 & 3 & 5.0 & 2.4 & 2.9 & -0.5 \\
\hline 22 & 30 & 3 & 5.0 & 2.4 & 2.3 & 0.1 \\
\hline 23 & 30 & 5 & 7.0 & 3.7 & 4.1 & -0.4 \\
\hline 24 & 30 & 5 & 7.0 & 3.7 & 5.6 & -1.9 \\
\hline 25 & 30 & 5 & 6.5 & 3.4 & 4.9 & -1.5 \\
\hline 26 & 30 & 5 & 6.5 & 3.4 & 3.3 & 0.1 \\
\hline 27 & 30 & 5 & 6.5 & 3.4 & 4.1 & -0.7 \\
\hline 28 & 30 & 5 & 5.0 & 2.6 & 2.3 & 0.3 \\
\hline 29 & 30 & 6 & 5.0 & 2.7 & 3.0 & -0.3 \\
\hline 30 & 30 & 6 & 5.0 & 2.7 & 2.8 & -0.1 \\
\hline 31 & 30 & 6 & 5.0 & 2.7 & 2.9 & -0.2 \\
\hline 32 & 30 & 6 & 5.0 & 2.7 & 3.0 & -0.3 \\
\hline 33 & 30 & 8 & 7.0 & 3.8 & 4.2 & -0.4 \\
\hline \multirow[t]{2}{*}{34} & 30 & 8 & 7.0 & 3.8 & 4.1 & -0.3 \\
\hline & & & Mean values & 3.0 & 3.1 & -0.1 \\
\hline
\end{tabular}


Table 3. Comparisons between calculated treatment planning system (TPS) rectal doses and doses in-vivo with respect to the minimum and maximum values in the thirty-four intracavitary brachytherapy (ICBT) insertions

\begin{tabular}{|c|c|c|c|c|c|c|c|c|}
\hline $\begin{array}{c}\text { Cylinder, } \\
\text { diameter }(\mathrm{mm})\end{array}$ & $\begin{array}{l}\text { Treatment, } \\
\text { length }(\mathrm{cm}) \\
\end{array}$ & $n$ & $\begin{array}{l}\text { Min. calculated } \\
\text { dose (TPS) (Gy) }\end{array}$ & $\begin{array}{c}\text { Min. in-vivo } \\
\text { dose (Gy) }\end{array}$ & Dev. (Gy) & $\begin{array}{l}\text { Max. calculated } \\
\text { dose (Gy) }\end{array}$ & $\begin{array}{c}\text { Max. in-vivo } \\
\text { dose (Gy) }\end{array}$ & Dev. (Gy) \\
\hline 25 & 2 & 2 & 2.0 & 1.5 & 0.5 & 2.1 & 2.9 & -0.8 \\
\hline 25 & 3 & 2 & 2.9 & 0.4 & 2.5 & 3.3 & 5.1 & -1.8 \\
\hline 30 & 3 & 2 & 2.2 & 0.4 & 1.8 & 2.5 & 5.7 & -3.2 \\
\hline 20 & 4 & 4 & 2.8 & 0.8 & 2.0 & 3.3 & 6.3 & -3.0 \\
\hline 25 & 4 & 6 & 2.2 & 0.5 & 1.7 & 2.5 & 5.3 & -2.8 \\
\hline 20 & 5 & 2 & 2.0 & 0.7 & 1.3 & 2.4 & 6.7 & -4.3 \\
\hline 25 & 5 & 4 & 3.1 & 2.2 & 0.9 & 3.6 & 7.0 & -3.4 \\
\hline 30 & 5 & 6 & 2.4 & 1.2 & 1.2 & 2.7 & 9.7 & -7.0 \\
\hline 30 & 6 & 4 & 3.2 & 1.1 & 2.1 & 3.7 & 7.5 & -3.8 \\
\hline \multirow[t]{2}{*}{30} & 8 & 2 & 3.4 & 1.1 & 2.3 & 4.1 & 9.1 & -5.0 \\
\hline & & & & $r=0.30$ & & & $r=0.55$ & \\
\hline
\end{tabular}

TPS - treatment planning system, ICBT - intracavitary brachytherapy, $n$ - number of brachytherapy insertions, dev. - deviation of in-vivo dose from the calculated value

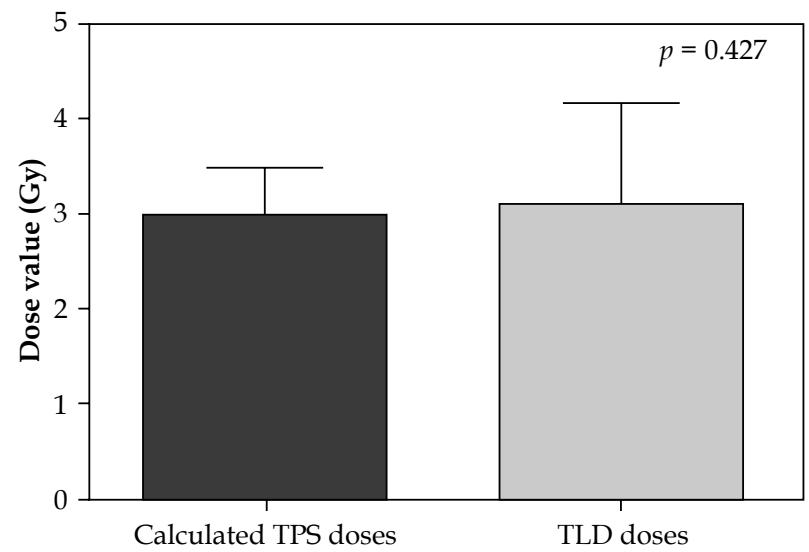

Fig. 3. Comparison of rectal calculated TPS dose values with TLD values in vaginal brachytherapy using cylinders. There is no significant difference between the calculated TPS doses and measured TLD doses $(p=0.427)$

TLD doses were obtained when $30 \mathrm{~mm}$ cylinder applicator was used for 5-8 $\mathrm{cm}$ treatment lengths as indicated in Table 3 . This confirmed the expected trend obtainable on the TPS where the mean calculated doses increased with cylinder size and the treatment lengths as indicated in Table 2. In a previous study, Demanes et al. [3] examined the use and advantages of a multichannel vaginal cylinder over the single-channel type in HDR brachytherapy. In our study, the vaginal cylinders produced the expected circular isodose distribution in the transverse plane, and the elongated oval shape in the AP and lateral planes as in the study by Demanes et al. The role of in-vivo dosimetry for cervical cancer patients treated with tandem and ovoids was evaluated in a study by Waldhäusl et al. [7]. Flexible probes from PTW/Germany were used. The rectal probes (type 9112) had five semiconductors (separated by $15 \mathrm{~mm}$ ) and the bladder probes (type 9113) had one.
The outer diameters were 7 and $3 \mathrm{~mm}$, respectively. The probes were connected to a computerized control system. For the rectum, the difference between the calculated and the measured dose varied between -31 and $90 \%$. Forty-four out of fifty-five ( $80 \%$ ) applications showed a higher calculated dose and only eleven out fifty five (20\%) applications showed a higher measured dose. The observed differences in the previous study were mainly attributed to probe movement during the time interval between radiographs and end of the irradiation. The results of the present work have shown deviations between -70.8 and 63.6\%. In TLD dosimetry, 14/34 (41.2\%) applications showed a higher calculated dose, 19/34 (55.9\%) indicated a higher measured dose and 1 (2.9\%) application showed no difference. In this case, movement of the rectal marker bearing the TLDs is minimal as the procedure did not involve radiographic imaging and the patients were not moved prior to treatment delivery. Table 2 shows that the minimum rectal TLD doses were lower than the corresponding TPS values with a weak correlation of 0.30 . Conversely, the maximum in-vivo doses, which were higher than the calculated TPS values showed a better correlation of 0.55 . The differences between doses measured in-vivo and the other method could be attributed to the slight intrinsic bend on the flexible rectal marker. Hence, part of the TLDs could be tilted inward or outward of the isodose line describing the dose distribution at the intended points of dose measurements. This would imply greater variations in measured rectal TLD doses for each brachytherapy insertions than as obtainable in TPS calculated values. The differences between the means of the calculated doses and the measured values were however found not to be statistically significant as depicted by Figure 3. This is also evident from the comparable overall mean doses of $3.0 \pm 0.5$ Gy and 3.1 \pm 1.1 Gy corresponding to 48.9 and $50.8 \%$ of the prescribed dose, respectively. The marked difference in their standard deviations could 
be attributed to two reasons. First, for a given application, the calculated doses at predefined points longitudinally around the cylinder on a treatment plan are more reproducible than doses measured in-vivo at R1-R5 along the rectal marker. Also, the mean calculated dose for a given brachytherapy insertion remains unchanged for a patient when the cylinder applicator size and treatment length are the same in subsequent treatment fractions. This is not the case in in-vivo measurements, where the mean dose for each treatment application is unique. The pliability of the re-usable rectal marker engendered a wider range of in-vivo doses as reflected in the mean dose (per insertion) varying from 1.2-5.6 Gy compared with 2.1-3.8 Gy for the TPS values presented in Table 2.

In $33(97.1 \%)$ of the applications, the measured doses, which varied considerably across the rectal TLDs were minimum at the innermost TLDs on the rectal marker. In other words, the in-vivo doses were minimum at the superior end (first dosimeter) of the rectal marker and the maximum values were mostly obtained at the central (third) dosimeter. This is in similitude to the pattern of dose distribution observed across the defined control points on the TPS (Table 1B). Maximum rectal TLD doses obtained in this study were associated with the use of $30 \mathrm{~mm}$ vaginal cylinder for treatment lengths of $5-8 \mathrm{~cm}$. But it was observed that the effect of the large applicator size on the in-vivo dose was reduced when the treatment length was $<4 \mathrm{~cm}$. Therefore, in ICBT, the choice of the largest cylinder and source-activated length $\geq 5 \mathrm{~cm}$ must be clinically justified before use. In this circumstance, wherever possible, individual patient treatment planning for each vaginal vault treatment insertion may be adopted using 3D images. Magnetic resonance imaging guidance allows treatment to be optimized to tumor volumes while sparing the organs at risk [11].

\section{Conclusions}

This study, being a part of efforts to conform with good brachytherapy practice in HDR vaginal vault treatment, provides a preliminary evaluation of the current status. There was no significant difference in the mean doses to the rectum between values derived from the planning system and that of in-vivo dosimetry. The study outcome is an indication of the quality of brachytherapy practice at the University College Hospital, Ibadan, Nigeria. However, wherever possible, image-based brachytherapy planning should be adopted for treatment in lieu of 2D standard plans in order to control extreme doses that may arise at some points within the rectum volume. In the modern era, the use of 3D imaging is paramount due to the close proximity of critical organs, which are underassessed with 2D planning [12].

\section{Acknowledgments}

The authors acknowledge the International Atomic Energy Agency (IAEA), Vienna, Austria for facilitating the availability of the first HDR brachytherapy equipment in Nigeria at our center. We sincerely thank Mr. Jan Hough and the staff of the engineering workshop of the
Department of Medical Physics, Groote Schuur Hospital, Cape Town, South Africa for producing the re-usable rectal marker used for this study. Also, we are grateful to the Senate, University of Ibadan, Ibadan, Nigeria for providing the research grant used in procuring TLD-100 rods and the Center for Energy, Research and Development (CERD), Ile-Ife, Nigeria for allowing the use of their TLD Reader and the oven.

\section{Disclosure}

Authors report no conflict of interest.

\section{References}

1. Sakata K, Nagakura H, Oouchi A et al. High-dose-rate intracavitary brachytherapy: results of analyses of late rectal complications. Int J Radiat Oncol Biol Phys 2002; 54: 1369-1376.

2. Nas S, Dally M, de la Torre M et al. Recommendations for implementation of high dose rate 192Ir brachytherapy in developing countries by the Advisory Group of International Atomic Energy Agency. Radiother Oncol 2002; 64: 297-308.

3. Demanes J, Rege S, Rodriquez RR et al. The use and advantages of a multichannel vaginal cylinder in high-dose-rate brachytherapy. Int J Radiat Oncol Biol Phys 1999; 44: 211-219.

4. Lahtinen $T$, Tenhunen $M$, Väyrynen $M$. ICRU reference points and maximum doses of rectum and bladder in intracavitary radiotherapy. Radiother Oncol 1993; 28: 174-176.

5. Clark B, Souhami L, Roman T et al. Rectal complications in patients with carcinoma of the cervix treated with concomitant cisplatin and external beam irradiation with high dose rate brachytherapy: a dosimetric analysis. Int J Radiat Oncol Biol Phys 1994; 28: 1243-1250.

6. Alecu R, Alecu M. In-vivo rectal dose measurements with diodes to avoid misadministration during intracavitary high dose rate brachytherapy for carcinoma of the cervix. Med Phys 1999; 26: 768-770.

7. Waldhäusl C, Wambersie A, Pötter R et al. In-vivo dosimetry for gynaecological brachytherapy: physical and clinical considerations. Radiother Oncol 2005; 77: 310-317.

8. Guler O, Onal C, Acibuci I. Effects of bladder distension on dose distribution of vaginal vault brachytherapy in patients with endometrial cancer. I Contemp Brachytherapy 2015; 6: 371-376.

9. International Commission on Radiation Units and Measurements: Dose and Volume Specification for Reporting Intracavitary Therapy in Gynecology, ICRU Report 38 (1985) ICRU, Bethesda, MD.

10. Baltas D, Sakelliou L, Zamboglou N (eds.). The Physics of Modern Brachytherapy for Oncology. Series in Medical Physics and Biomedical Engineering. Taylor \& Francis Group, Boca Raton 2007.

11. Anderson J, Huang Y, Kim Y. Dosimetric impact of point A definition on high-dose-rate brachytherapy for cervical cancer: evaluations on conventional point A and MRI-guided, conformal plans. J Contemp Brachytherapy 2012; 4: 241-246.

12. Glaser S, Beriwal S. Brachytherapy for malignancies of the vagina in the 3D era. J Contemp Brachytherapy 2015; 7: 312-318. 